\title{
Precursors of social emotional functioning among full-term and preterm infants at 12 months: Early infant withdrawal behavior and symptoms of maternal depression
}

Vibeke Moe, Hanne Cecilie Braarud, Tore Wentzel-Larsen, Kari Slinning, Unni Tranaas

Vannebo, Antoine Guedeney, Mikael Heimann, Anne Margrethe Rostad and Lars Smith

\section{Journal Article}

\section{Tweet}

N.B.: When citing this work, cite the original article.

Original Publication:

Vibeke Moe, Hanne Cecilie Braarud, Tore Wentzel-Larsen, Kari Slinning, Unni Tranaas Vannebo, Antoine Guedeney, Mikael Heimann, Anne Margrethe Rostad and Lars Smith, Precursors of social emotional functioning among full-term and preterm infants at 12 months: Early infant withdrawal behavior and symptoms of maternal depression, Infant Behavior and Development, 2016. 44, pp.159-168.

http://dx.doi.org/10.1016/j.infbeh.2016.06.012

Copyright: Elsevier

$$
\text { http://www.elsevier.com/ }
$$

Postprint available at: Linköping University Electronic Press

http://urn.kb.se/resolve?urn=urn:nbn:se:liu:diva-131698

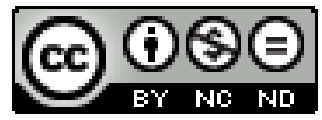


I..U 
Title: Precursors of social emotional functioning among full-term and moderately preterm infants at 12 months: Early infant withdrawal behavior and symptoms of maternal depression.

Vibeke Moe $^{1,2}$, Hanne Cecilie Braarud ${ }^{2,3}$, Tore Wentzel-Larsen ${ }^{4,5}$, Kari Slinning ${ }^{2,1}$, Unni

Tranaas Vannebo ${ }^{2}$, Antoine Guedeney ${ }^{6}$, Mikael Heimann ${ }^{7}$, Anne Margrethe $\operatorname{Rostad}^{8}$, and Lars Smith $^{1}$

1. Department of Psychology, University of Oslo, Norway

2. National Network for Infant Mental Health, the Regional Center for Child and Adolescent Mental Health, Eastern and Southern Norway, Oslo, Norway

3. Regional Centre for Child and Youth Mental Health and Child Welfare West, Uni Research Health

4. Norwegian Center for Violence and Traumatic Stress Studies, Oslo, Norway

5. The Regional Center for Child and Adolescent Mental Health, Eastern and Southern Norway, Oslo, Norway

6. Department of Child and Adolescent Psychiatry, Hospital Bichat-Claude Bernhard APHP, France and Université, Paris 7, France

7. Division of Psychology, Department of Behavioural Sciences and Learning, Linköping University, Sweden

8. Municipality of Trondheim's Welfare Clinic, Norway

Corresponding author:

Vibeke Moe, Department of Psychology, University of Oslo, POB 1094, 0317 Oslo, Norway.

Email: vibeke.moe @psykologi.uio.no

Phone: +4792850019 


\title{
Precursors of social emotional functioning among full-term and preterm infants at 12 months: Early infant withdrawal behavior and symptoms of maternal depression.
}

\begin{abstract}
This study forms part of a longitudinal investigation of early infant social withdrawal, maternal symptoms of depression and later child social emotional functioning. The sample consisted of a group of full-term infants $(\mathrm{N}=238)$ and their mothers, and a group of moderately premature infants $(\mathrm{N}=64)$ and their mothers. At 3 months, the infants were observed with the Alarm Distress Baby Scale (ADBB) and the mothers completed the Edinburgh Postnatal Depression Scale (EPDS). At 12 months, the mothers filled out questionnaires about the infants' social emotional functioning (Infant Toddler Social Emotional Assessment and the Ages and Stages Questionnaire-Social Emotional). At 3 months, as we have previously shown, the premature infants had exhibited more withdrawal behavior and their mothers reported elevated maternal depressive symptoms as compared with the full-born group. At 12 months the mothers of the premature infants reported more child internalizing behavior. These data suggest that infant withdrawal behavior as well as maternal depressive mood may serve as sensitive indices of early risk status. Further, the results suggest that early maternal depressive symptoms are a salient predictor of later child social emotional functioning. However, neither early infant withdrawal behavior, nor gestational age, did predict social emotional outcome at 12 months. It should be noted that the differences in strength of the relations between ADBB and EPDS, respectively, to the outcome at 12 months was modest. An implication of the study is that clinicians should be aware of the complex interplay between early infant withdrawal and signs of maternal postpartum depression in planning ports of entry for early intervention.
\end{abstract}


Keywords: Infant social withdrawal, maternal depressive symptoms, full-term and preterm infants, social emotional functioning 


\section{Precursors of social emotional functioning among full-term and preterm infants at 12 months: Early infant withdrawal behavior and symptoms of maternal depression.}

\section{Introduction}

At one year of age, infants usually show a distinctive pattern of social emotional behaviors. At this age level the primary regulation pattern of approach and avoidance has differentiated into the early emotions of interest, joy, surprise, sadness, anger, disgust and fear (Izard, Huebner, Risser, McGinness, \& Dougherty, 1980; Lewis, 2014). These early emotions are difficult to capture in a snapshot, but are more conspicuous when a parent is asked to describe the infant's predominant emotional functioning in a social context. There is, however, a dearth of knowledge of the precursors of infant social emotional functioning around the first birthday. This paper takes a look at the subdued side of a conjectural precursory mechanism: infant sustained withdrawal behavior at age 3 months and its maternal counterpart, i.e., the mothers' concurrent self-reported depressive symptoms.

The question of how early in infancy one may identify possible precursors of later child social emotional development is central in developmental psychopathology. One may ask if there is an association between early infant affective reactivity and maternal emotional functioning as observed when the baby is about 3 months old, and infant social emotional behavior nine months later, at a time when the emotions have become integrated parts of action patterns tied to particular situations. A related question concerns the saliency of infant versus parental factors when looking for an association between possible predictors very early in the first year of life and later child development. For example, it has been found that symptoms of post-partum depression affect 10-15 percent of mothers in the first months after birth (Eberhard-Gran, Slinning, \& Rognerud, 2014; O’Hara \& Swain, 1996), and that infants exposed to maternal life stressors and depressive symptoms in the first months of life are at 
risk for early social withdrawal (Braarud et al. 2013; Burtchen et al., 2013; Guedeney, Marchand-Martin, Cote, Larroque, \& the EDEN Mother-Child Cohort Study Group, 2012) and adverse social emotional development later (Goodman et al., 2011). Further, Stein et al. (2014) in a review of the recent literature showed that maternal depression and other mental disorders in the perinatal period are associated with increased risk of psychological and developmental disturbances in children. More related to maternal depression specifically, Goodman et al.'s meta-analytic review (2011) examined the strength of the association between mothers' depression and children's behavioral problems or emotional functioning. They found that maternal depression was significantly related to an array of later adverse social emotional development, such as internalizing and externalizing behavior. However, none of these studies reported on the relative importance of early infant withdrawal versus maternal postpartum depressive symptoms to later social emotional functioning.

Together with maternal postpartum depressive symptoms, a variety of factors may be associated with social withdrawal behavior in infancy, such as malnutrition, childhood abuse, mental retardation, intrauterine alcohol exposure, autism spectrum disorders and prematurity. Infant social withdrawal may also be associated with temperamental dispositions, relationship difficulties, attachment disorders and parental mental health issues (cf. Guedeney \& Fermanian, 2001; Guedeney, Matthey, \& Puura, 2013). Rather than constituting a diagnosis, social withdrawal might be viewed as an alarm signal for both organic and relationship disorders. In order to provide a systematic means to assess withdrawal behavior in infants aged 2-24 months, Guedeney and Fermanian (2001) constructed and validated an observation method, the Alarm Distress Baby Scale (ADBB).

Studies of the validity of ADBB have shown that the scale differentiates between clinic referred and non-referred infants (Dollberg, Feldman, Keren, \& Guedeney, 2006), and between full-term and premature infants (Braarud et al, 2013). Braarud and colleagues (2013) 
found that premature infants had significantly higher ADBB composite scores at 3 and 6 months of age (but not at 9 months), as compared with full-term infants. In the large population-based EDEN-study it was found that preterm birth and low birth weight were associated with social withdrawal at 12 months. Social withdrawal was also associated with maternal and paternal vulnerabilities (Guedeney et al., 2012).

In the Braarud et al. study (2013) a significant relation between maternal depressive symptoms at 3 and 6 months, and infants' social withdrawal at 9 months was found in the full-term group. It should also be noted that concurrent associations between the two measures were found at 6 and 9 months. In a study by Matthey et al. (2005) infants 3-12 months old were observed during a routine physical examination; ADBB scores were obtained from videotaped interactions with a health professional. In addition, the parents' self-reported depressive symptoms and perceived mental health since birth were obtained. It was found that mothers who reported feelings of irritability, anxiety and sadness had infants with significantly higher ADBB scores, as compared with mothers who did not report such feelings. However, Matthey and colleagues observed that infant social withdrawal was associated significantly only with mothers' retrospective reports of mood difficulties since birth, not with concurrent depressive symptoms as assessed on the Edinburgh Postnatal Depression Scale (EPDS; Cox, Holden, \& Sagovsky, 1987).

Longitudinal stability has been an issue in many areas of developmental research, but few studies have been able to establish a firm connection between infant withdrawal and later behavioral phenotypes. Therefore, in the present context it is of special interest that this question was investigated in a study of the longer-term impact of social withdrawal among infants (Milne, Greenway, Guedeney, \& Larroque, 2009). Infants were assessed for social withdrawal at 6 months. Participants were seen again at age 30 months, at which time the children were assessed on Bayley-III (Bayley, 2005), and the mothers completed a 
questionnaire about their child's social emotional behavior with the Behavior Assessment System for Children (Reynold \& Kamphaus, 2004). The results showed that infant withdrawal behavior was associated with maternally reported atypicality and attention problems at 30 months. Milne et al. (2009) also reported that infants' ADBB scores were negatively related to later cognitive and language Bayley scores.

The present report forms part of a longitudinal study from 3 to 12 months of early infant social withdrawal, maternal symptoms of depression and later child social emotional functioning in a mixed sample of low-risk and premature children (see also Braarud et al., 2013). Two groups of infants (full-term versus moderately preterm) and their mothers, who were seen repeatedly during the child's first year of life, participated.

The purpose of the present paper is twofold: First, to analyze possible group differences in social emotional behavior at 12 months between full-term and preterm infants. Second, to present longitudinal data from 3 to 12 months in order to analyze the contribution of early infant social withdrawal behavior and maternal symptoms of depression to subsequent child social emotional behavior.

It was expected that mothers of the premature infants would report more child social emotional difficulties, especially internalizing problems, at 12 months of age, compared to mothers of full-term infants. Furthermore, in line with the transactional model positing a continuous and reciprocal influence between infant and caregiver over time, making each participant an active partner in the interaction (Sameroff, 2010), we hypothesized that early infant social withdrawal and maternal symptoms of depression would each make an independent contribution to later child social emotional functioning. That is, we assumed that each of these two variables would contribute to the prediction from 3 to 12 months when the infant gestational age and maternal education was controlled for. 


\section{Method}

\section{Participants and recruiting}

The total sample consisted of a group of full-term infants $(\mathrm{N}=238)$ and their mothers, and a group of premature infants born gestational week 30-36 $(\mathrm{N}=64)$ and their mothers. The recruitment took place in the municipality of Trondheim, the third largest city in Norway. The participants in the full-term group were enrolled from well-baby clinics in the four districts that collaborated in the project, while all well-baby clinics in Trondheim contributed to enrollment of the premature group. Mothers were informed about the study at the regular home visit shortly after birth and received an information leaflet about the study. The families who volunteered for the study were asked to give a written consent about participation at the regular health check-up when their infant was 6 weeks old. The study consisted of a community sample, and the mothers had to speak Norwegian fluently and be motivated to participate at all follow-up assessments of the study (i.e., at infant ages 3-, 6-, 9- and 12 months, but only data at 3-and 12 months were included in the present report). No other exclusion criteria were applied for the mothers. Further, to be eligible to participate in the study the infants should have a gestational age between 30 and 42 weeks (the premature infants30-36 weeks, the full-term infants 37-42 weeks).Exclusion criteria for the infants were acute and chronic neurological problems, known medical diagnoses, visual or auditory impairment, and small for gestational age (birth weight below the third standard deviation on pediatric growth charts).

The present study includes data from medical birth records and data collected from the assessments when the infants were 3 and 12 months of age (corrected age for the premature infants). At 3 months 300 (62 prematures) infants were observed with the ADBB scale. At the same age point 282 mothers (61 mothers of prematures) completed EPDS. No dyads had 
missing data on both of these measures. At 12 months 246 mothers (56 mothers of prematures) submitted data by filling out questionnaires, $(79.8 \%$ of the full-term group and $90.3 \%$ of the premature group) Since some mothers did not answer the complete set of questions in the inventories, the number of participants varies somewhat between the different outcome measures employed at 12 months. In these cases, the exact numbers were determined by the calculation rules specified in the manuals.

The research protocol was approved by the Regional Ethical Committee for Medical Research in Eastern and Southern Norway and The Data Protection for Official Research.

\section{Background information.}

Information about birth weight and gestational age (GA) was collected from the infants' medical birth records; the mothers gave information about their age and educational level.

\section{Measures at 3 months}

The Alarm Distress Baby Scale. The Alarm Distress Baby Scale (ADBB) is a clinical method aimed at evaluating social behaviors that can be easily observed during a brief observation of children 2-24 months old (Guedeney \& Fermanian, 2001). These behaviors are organized into eight items: (1) facial expression, (2) eye contact, (3) general level of activity, (4) selfstimulating gestures, (5) vocalizations, (6) response to stimulation, (7) relationship, and (8) attraction. Each item is rated on a scale from 0 to $4(0=$ no unusual behavior; $4=$ severe unusual behavior), the lowest possible composite score is 0 and the highest 32 . A trained observer needs only an observation of 10 to 15 minutes in order to score ADBB (Guedeney, personal communication). Guedeney and Fermanian (2001) used a cut-off score of five and reported a sensitivity of .82 , a specificity of .78 . They reported Cronbach's alpha for the ADBB scale to be 0.83 . In the present study, ten public health nurses and one pediatrician participated as ADBB raters for the full-term group (the pediatrician participated in the 
reliability training, but only contributed to the data collection at 6 months, not included in the present study), and two professionals (one experienced health nurse and one specialist in clinical child psychology (authors UTV and AMR) participated as ADBB raters for the premature group. All raters were experienced professionals and the majority had worked for many years in well-baby clinics.

The reliability training was twofold. The public health nurses and the pediatrician who scored the full-term cohort took part in a 2-day training held by Antoine Guedeney (A.G). Then, in the following 4-month period, each participant received individual training tapes to practice on, and they met five times as a group to discuss and evaluate their scorings with the supervision team (S-team). Two professionals (authors U.T.V and A.M.R) scored the premature cohort. They were trained to reliability by A.G, with three other professionals (authors V.M, H.C.B, and K.S) from the S-team. Interrater reliability was calculated for all raters before the project started. The public health nurses scored seven video clips while the pediatrician scored four clips. Agreement on caseness ( $\geq 5$ ) was acceptable (89 \% agreement; Cohen's $\kappa=.78$ ) for the public health nurses and the pediatrician. The same criterion for caseness was used for the S-team. In addition, and because of their status as supervisors, there should be no more than 1-point rating difference per case when compared to the video clips provided for interrater reliability assessment. Ratings from each rater were entered in a $4 \times 4$ contingency table. The calculation of Cohen's $\kappa$ for the S-team on four video clips gave a satisfactory $\kappa(\kappa=.80-.96)$. Since two of the raters made one rating with more than 1 -point difference on one video clip each, four of the S-team members rated six more video clips which yielded a satisfactory $\kappa(\kappa=.92-1.0)$ Members of the S-team met with public health nurses and the pediatrician on a regular basis during the data collection period. 
The Edinburgh Postnatal Depression Scale. The Edinburgh Postnatal Depression Scale (EPDS) (Cox, Holden \& Sagovsky, 1987) is a self-report instrument of 10 items designed to assess depressive symptoms during the last seven days. Each item is rated on a 4-point Likert scale (0-3) to produce a composite score ranging from 0 to 30 , with higher scores indicating elevated risk for postpartum depression. Compared with other measures of depressive symptoms, EPDS does not consist of items that are common to nearly all new mothers, such as loss of energy, feeling tired, changes in appetite and sexual drive. EPDS has been validated in two Norwegian studies and has shown high sensitivity and specificity with cut-off score $\geq 10$ (Eberhard_Gran, Eskild, Tambs, Schel, \& Opjordsmoen, 2001) and $\geq 11$ (Berle, Aarre, Mykletun, Dahl, \& Holsten, 2003), respectively. Eberhard Gran et al. (2001) reported good internal consistency (Cronbach's alpha $=.81)$.

\section{Measures at 12 months}

The Ages and Stages Questionnaires - Social Emotional (ASQ:SE). ASQ:SE is a screening instrument tapping the areas of self-regulation, compliance, communication, adaptive autonomy, affect and interaction with people (Squires, Bricker, \& Twombly, 2002). The questionnaire at 12-months contains 25 items and takes 10-15 minutes for parents to complete. Some examples are: "Does the child laugh or smile at you or other family members?", “Does your baby look for you when a stranger approach?”, "Does your baby stiffen and arch her back when picked up?", "Does your baby cry, scream or have tantrums for long periods of time?" Each item is scored "most of the time", "sometimes", "rarely or never, and "of concern?" Composite scores may extend from 0 to 220 (330 if the "of concern" questions are included). At 12 months the cut-off point is 48 . This instrument was developed in the US and has been translated to Norwegian. The reported Cronbach's alpha for a Swedish validation of the ASQ: SE is .79 (Salomonsson \& Sleed, 2010). 
The Infant-Toddler Social and Emotional Assessment. The Infant-Toddler Social and Emotional Assessment (ITSEA) is a parent reported questionnaire comprising 166 items measuring four domains (externalizing, internalizing, dysregulation, and competence; each domain expressed as a T-score value) (Carter, Littler, Briggs-Gowan, \& Kogan, 1999).

The externalizing domain comprises measures of activity /impulsivity, aggression/defiance, and peer aggression. The internalizing domain includes depression/ withdrawal, general anxiety, separation distress, and inhibition to novelty. The dysregulation domain contains negative emotionality, sleep, eating, and sensory sensitivity. Finally, the domain "Competence" assesses socio-emotional capabilities. This domain was excluded for the present purposes since the aim of the study was to relate early assessment of withdrawal behavior and maternal depressive symptoms to later signs of aberrant child behaviors.

In order to calculate a T-score for each domain, a specified number of questions had to be answered according to the manual. Thus in the present study $\mathrm{N}$ varied somewhat between the different domains. T-scores $\geq 1.5$ standard deviation $(\geq 65)$ at or above the mean on the Externalizing, Internalizing and/or Dysregulation are considered to be in the "Of Concern" range. Responses on the ITSEA are rated on a 3-point scale (not true/rarely; somewhat true/sometimes; and very true/often); it takes approximately 30 minutes to complete the questionnaire. Carter, Briggs-Gowan, Jones, and Littler (2003) reported good internal consistency for ITSEA domains (Cronbach's alpha range: .80 to .90). In the present study the Norwegian translation was used.

\section{Procedure}

All infants were followed longitudinally and assessed at 3, and again at 12 months of age. For the full-term infants the examinations at 3 months were carried out during the regularly scheduled visits to the well-baby clinics. For reliability purposes all examinations were taped 
with a video camera by a person present in the room. The necessary information for scoring ADBB was obtained during these routine examinations. The examinations were performed by the public health nurses. Thus, no extra examination that might burden the child or mother was needed.

For the premature infants the examinations at 3 months were carried out as an extra observation at the well-baby clinics or during the regularly scheduled visits by two of the specialists in the research team. Infants with severe withdrawal behavior or other significant symptoms of developmental delay or impairments were either invited to a follow-up consultation and further assessment or referred, if necessary, for further evaluation and treatment. In addition to the examinations of the infants, the mothers filled out EPDS at 3 months postpartum. The public health nurses collected background information from the infant's birth register form, such as the infant's medical status at birth, gestational age and birth weight, and the parents' age and education.

The outcome measures at 12 months, ITSEA and ASQ: SE, were mailed to the families and completed in advance or at a visit to the well-baby clinic.

\section{Statistical methods}

The two groups were compared on infant gestational age and birthweight, maternal age, maternal EPDS score and infant ADBB score, both administered at 3 months, using independent t-tests checked with permutation t-tests in case of marked skewness. For categories of maternal educational level, the differences between the groups were analyzed by exact chi-square tests. Independent t-tests were also conducted to examine the differences between groups on the outcome variables (Externalizing, Internalizing and Dysregulation domains from the ITSEA, and ASQ: SE). 
To check whether the independent and control variables at 3 months could be employed as separate independent and control variables in the regressions analyses, intercorrelations between these variables were computed using Pearson product-moment correlation coefficients (for continuous variables) and Spearman rank order correlation (for categorical variables) coefficients. In addition, variance inflation factors (preferably less than 10) were computed in all regression models.

In order to analyze the differential contribution of infant social withdrawal behavior and maternal depressive symptoms to subsequent child social emotional behavior, four linear regression analyses were conducted with ITSEA externalizing, internalizing, and dysregulation domains, and ASQ: SE as dependent variables. The following independent variables were entered: maternal symptoms of depression at infant age 3 months (total EPDS score) and signs of infant social withdrawal at 3 months (total ADBB score). Infant gestational age (GA), maternal level of education and maternal age were used as control variables. Relations with EPDS and ADBB in each regression, per $10 \%$ of the total possible range within the scales of EPDS and ADBB, were compared by testing of linear hypotheses within each regression.

The variables analyzed in this study were both continuous and categorical. Level of education (categorized as low to high from level 1 to 4), was treated as a categorical variable, while maternal age and infant gestational age were treated as continuous variables. All outcome variables were continuous measures. Significant results were determined by $p<.05$. Variance explained was specified as (adjusted) $\mathrm{R}^{2}$ values. The SPSS Version 22 (IBM SPSS Statistics 22) was used to perform most statistical analyses, while testing of linear hypotheses used the R (The R Foundation for Statistical Computing, Vienna, Austria) package car. 


\section{Results}

Descriptive statistics for each separate group and group differences

With respect to maternal educational level the group difference were close to statistically significant (mothers of full-term infants tended to have higher education, $\chi^{2}=7.22, p=$ $0.065)$.

As expected, and due to inclusion criteria, the two groups (full-terms and prematures) differed on gestational age and birth weight (see table 1). As shown in Table 1, there were no significant group differences in maternal age. At 3 months of age the premature infants had significantly higher mean total ADBB score, as compared with the full-term infants $(11.3 \%$ of the premature infants scored at or above the cut-off score $\geq 5$, while $4.6 \%$ of the full-term infants did so). Also, mothers of the premature infants reported a significantly higher mean total EPDS score than did mothers of the full-term infants $(23.0 \%$ of the mothers of the premature infants, and $5.9 \%$ of the mothers of the full-term infants scored at or above the cutoff $\geq 10$ ) (cf. also Braarud et al., 2013).

At 12 months of age the premature children evinced significantly higher mean ITSEA internalizing behaviour than did the full-term children. No significant group differences were found on the other two ITSEA domains (Externalizing or Dysregulation) or on ASQ: SE at 12 months postpartum (see Table 1).

\section{Insert Table 1 here}

In the ITSEA internalizing domain, five of $182(2.8 \%)$ of the full-term children, and one child of 56 (1.8\%) of the premature children, had scores in the "Of Concern" range. In the ITSEA externalizing domain the corresponding numbers were nine of 178 (5.1\%) of the fullterm children, and one of $56(1.8 \%)$ in the premature group. In the ITSEA dysregulation domain the number of children with "Of Concern" scores were eight of $185(4.3 \%)$ of the full-terms, and two of 56 (3.6 \%) of the preterms. Finally, on ASQ:SE five of 190 (2.6 \%) of 
the full-term children, and none of the premature children scored above the cut-off value of 48.

\section{Descriptive statistics for the two groups combined}

Table 2 shows descriptive statistics for the dependent variables for the two groups combined (three ITSEA domains; [Externalising, Internalising and Dysregulation], and ASQ: SE, all assessed at 12 months postpartum), independent variables (ADBB and EPDS, assessed at 3 months postpartum) and control variables (gestational age, maternal education and age). As shown, the infants' mean ADBB scores and the mothers' mean EPDS scores tended to be rather low and well below the respective cut-off points. The different ITSEA domains were all somewhat below the expected mean T-score of 50, and the mean ASQ: SE score was below the normed cut-off value at 12 months.

\section{Insert table 2 here}

Intercorrelations between the independent and the control variables for the two groups combined

There was no significant association between the independent variables of infant social withdrawal and maternal signs of depression at 3 months. For the mothers, an expected significant positive association between age and level of education was found $\left(r_{s}=.38, p<\right.$. 01). Also, as expected, a significant positive association was found between gestational age and birthweight $(r=.78, p<.001)$. A significant negative association was found between maternal EPDS score and infant gestational age $(r=-.17, p=0.006)$, between infant ADBB score and gestational age $(r=-.33, p<.001)$, and between birthweight and ADBB score $(r=-$ $.23, p<.001)$.

It should be noted that the two independent variables (ADBB and EPDS at 3 months postpartum) were not significantly related, and that most of the correlations between the 
control variables were either statistically insignificant or low. Therefore, there should be no concern to use them as separate independent or control variables in the regression analyses. However, since infant gestational age and birthweight were highly correlated, it was decided to exclude birthweight from further analyses.

Multiple linear regression analyses of the relation between the independent variables and the four outcome variables (all assessed at 12 months postpartum) In the multiple regression analyses all variance inflation factors were $\leq 2$ (see table 3 ). The multiple linear regression analysis showed that only EPDS at 3 months postpartum had significant independent relations to the three ITSEA domains, when controlling for the contribution of infant gestational age, maternal age and education (see Table 3). ADBB at 3 months postpartum was not significantly related to the ITSEA outcome measures $(p \geq 0.218)$. The adjusted explained variance of the ITSEA Externalizing domain model was 18 percent, whereas it was 5 percent for the Internalizing domain model, and 1.7 percent for the Dysregulation domain model. With ASQ: SE as the outcome variable, a similar result was found, only EPDS had a significant independent contribution. In the ASQ: SE model, the adjusted explained variance was 0.7 percent. Again, ADBB was not significantly related to the prediction of the outcome variable.

As explained in the methods section above, in order to compare the relative strength of the relations, linear hypotheses were used. For the ITSEA externalizing domain the coefficient of EPDS was significantly larger than the (actually negative) coefficient of ADBB, both coefficients transformed to relate to a difference of $10 \%$ of the possible range $(p=0.030)$. However, $\mathrm{p} \geq 0.063$ in similar comparisons for the other two ITSEA scores and the ASQ: SE score. 


\section{Discussion}

In this study we analyzed possible group differences between full-term and moderately premature infants in social emotional behavior at 12 months. In addition, we examined the contribution of infant social withdrawal behavior and maternal depressive symptoms at 3 months postpartum to subsequent child social emotional behavior at 12 months. It was hypothesized that mothers of premature infants would report more child social emotional difficulties, especially internalizing problems, at 12 months of age, as compared with mothers of full-term infants. Further, we hypothesized that early infant social withdrawal and maternal depressive symptoms each would make an independent contribution to later child social emotional functioning.

As previously shown by Braarud et al. (2013), the premature infants evidenced more social withdrawal behavior at age 3 months, as compared with the full-term infants. Further, and in line with our first hypothesis, mothers perceived the premature infants as more internalizing on the Infant Toddler Social Emotional Assessment (ITSEA) nine months later, at 12 months. The present results concur with the literature, suggesting that prematurity is associated with early withdrawal in infancy (Braarud et al, 2013), as well as with internalizing difficulties in later childhood (e.g. Sykes et al., 1997; Tessier, Nadeau, Boivin, \& Tremblay, 1997). Some studies of premature children have mainly reported internalizing behavior problems (e.g. Aarnoudse-Moens, Weisglas-Kuperus, van Goudoever, \& Oosterlaan, 2009; Schothorst \& van Engeland, 1996), whereas other studies have found higher rates of both internalizing and externalizing problems (Bhutta, Cleves, Casey, Cradock, \& Anand, 2002). It is noteworthy that more recent research has shown that preschool depressive symptoms, such 
as internalization, may lead into a path of further depression in later childhood and early adolescence (cf. Luby, Gaffrey, Tillman, April, \& Belden, 2014).

As is well-known, parental interaction with premature infants tends to be disrupted because the infants do not give clear signals when they want to pause, when they have had too much stimulation or not enough. Parents, in turn, may avoid or overstimulate the baby, further worsening the infants' behavior or their own frustration (Field, 1977). Although the present study did not comprise observation of mother-infant interaction, there are good reasons to expect that the regular social encounters taking place between our socially withdrawn premature infants and their mothers often had been compromised. Other studies of early social withdrawal confirm that mothers with socially withdrawn infants tend to be less sensitive and feel less happy than mothers of non-withdrawn infants, and that the overall quality of mother-infant interaction is non-optimal (Costa \& Figueiredo, 2011). This is consistent with the findings of Puura, Guedeney, Mäntymaa, and Tamminnen (2007), who found that withdrawn infants showed less optimal performance during interaction with their mothers as compared with infants who were not socially withdrawn. In the present context it is also of interest to note that mother-child interaction observed as early as age 3 months has been found to be predictive of internalizing symptoms reported at $4 \frac{1}{2}$ years and again at 8 years of age (Gerhold, Laucht, Texdorf, Schmidt, \& Esser, 2002).

Our second hypothesis concerned the question of possible early precursors of later child social emotional functioning at age 12 months. The findings were based on an analysis of the total sample, where the two groups of full-term and moderately premature infants had been combined. The main observation was that despite low average EPDS scores, an antecedent was found in the mothers' postnatal emotional state; i.e., maternal symptoms of depression at 3 months, but that neither early infant withdrawal behavior (ADBB), nor gestational age, was significantly related to later child social emotional functioning (i.e. all 
three ITSEA domains and ASQ:SE). The results showed that although the premature infants (as previously shown) had evidenced more withdrawal behavior than the full-term infants at 3 months, no infant characteristics bore a significant relation to ITSEA or ASQ:SE areas of functioning at age 12 months. The lack of an association between ADBB scores at 3 months and later social emotional functioning might partly be explained by the fact that most of the present infants evidenced low amounts of withdrawal behavior (see also Braarud et al., 2013, reporting that only $4.6 \%$ in the full-term group and $11.3 \%$ in the premature group had an ADBB score at or above the cutoff point of 5 at 3 months), and that none (not even the premature infants) were at high biomedical risk (for instance, none of the children were extremely premature or had a very low birth weight). Our finding that having symptoms of social withdrawal at 3 months does not necessarily lead to aberrant child outcome later might be seen as a rather encouraging result. It is noteworthy, however, that even if the regression analyses had shown that only EPDS was significantly related to all outcome measures, when we compared the relative strength of the relations between ADBB and EPDS to the outcome at 12 months, a significantly stronger association with EPDS than with ADBB was found only for the ITSEA externalizing domain. Actually all relations of ADBB with the ITSEA domains were, contrary to expectation, negative. This may be accidental, and none of these relations reached significance.

Why do early maternal depressive symptoms seem to be a factor accountable for prediction? In the present study the infants' social emotional functioning at 12 months was based on maternal assessment of the behavior of their own infants. Therefore, the association between early maternal depressive symptoms and later child internalizing might have been influenced by some mothers' depressive perception of their infants. However, in the Braarud et al. study (2013) based on the same sample, maternal depressive symptoms had decreased from 3 to 9 months and had a rather low prevalence in both groups of mothers at 9 months. 
Thus, it is probable that the prevalence of maternal depressive symptoms remained low at 12 months. An alternative interpretation might be that early maternal depressive symptoms at 3 months, via numerous subsequent mother-infant interactions during the first year of life might have given rise to the observed lagged relation to later child internalizing at 12 months.

It is now well established that early maternal depression may place infants at higher risk for later child behavior problems, and in several studies a range of adverse childhood behavioral and emotional outcomes have been documented. This was shown by Stein and colleagues (2014) in a review of maternal depression and other mental disorders in the perinatal period and their associations with later psychological and developmental disturbances in children. In another meta-analysis, Goodman and colleagues (2011) found that maternal depression was significantly related to both higher levels of internalizing and externalizing behavior, as well as to general psychopathology and negative affect in children. Importantly, the mean age of children in the samples studied was negatively related to the magnitude of the effect sizes of the relation between maternal depression and children's later social emotional problems. The younger the mean age of the children studied, the stronger the effects of the associations. Goodman et al. (2011) point out that their results are consistent with the notion of a sensitive period, and that younger children are more vulnerable to the effects of maternal depression than older ones. Thus, it is of interest to observe that the infants who took part in the present study, and were exposed to maternal depressive symptoms as early as 3 months, later were reported to be susceptible to internalizing behavior.

Goodman and colleagues further pointed out that the majority of the studies in their review did not evidence causal processes. Even if there were significant associations between maternal depression and child outcome, the direction of the effects remains elusive. In order to understand the impact of maternal depression it is therefore important to consider infants' effects on mothers, and transactional processes over time as well (Goodman, 2007; Sameroff 
\& MacKenzie, 2003). Interactional disturbances between depressed mothers and their infants, i.e., less sensitivity of the mothers and less responsivity of the infants, have been found across many studies (Field, 2010). Mothers with symptoms of depression often display less positive facial expressions, fewer vocalizations, and tend to look at and touch their infants less. The infants, in turn, show fewer positive facial expressions and vocalizations, more gaze aversion, and lower activity levels (cf. Field, 1995; 2010). These infant behaviors are similar to the items emphasized in the Alarm Distress Baby Scale (e.g., facial expression, eye contact, general level of activity, vocalizations, and relating). Burtchen and colleagues (2013), in a sample of clinically depressed mothers, showed that those with major depression tended to have infants who scored higher on withdrawal behavior, based on videotaped mother-infant interactions. A caveat should be stated: it is probably not the diagnosis of depression per se that predicts the quality of mother-infant interaction, but rather maternal ability to be sensitive and responsive to the infant's signals despite a depressive state (cf., Campbell, Cohn, \& Meyers, 1995). Moreover, as suggested by Costa and Figueiredo (2011, 2013), mothers of withdrawn infants may be less sensitive and feel less happy than mothers of more extroverted infants, but the direction of effects still remains unclear.

\section{Limitations}

As mentioned above, one limitation of the present study may be the possible bias of using maternally reported measures of child social emotional functioning. While professionals rated the infants' withdrawal behavior at 3 months, children's social emotional functioning at 12 months postpartum was based on mothers' response to questionnaires. The associations between early infant social withdrawal behavior and later social emotional behavior might have been stronger if the 12-month assessments had been based on behavioral observations by professionals, or on professionals' responses to ITSEA and ASQ:SE. 
Maternal depressive symptoms at 3 months post partum were also measured by a selfreport measure. One limitation of using a self-report instead of a diagnostic procedure is that the mothers might have underreported the presence of depressive mood. Maternal depressive symptoms at 12 months were not included in the present study since our aim was to study how early assessment of maternal depressive symptoms and infant withdrawal behavior (both measured at 3 months) might be related to later child social emotional functioning. As mentioned above, it is still a possibility that maternally reported child socio emotional functioning was influenced by maternal depressive mood and might have been influenced by the mothers' perception of their infants at 12 months.

Another limitation is related to the sample characteristics. The infants who participated in the study showed relatively low amounts and modest variance with respect to social withdrawal behavior. Further, as mentioned above, the premature infants were at relatively low biomedical risk. This may further have contributed to reduced statistical power and therefore might have increased the uncertainty of the estimated relations between the independent and the dependent variables.

\section{Conclusions}

In a previous study we showed that the premature infants evinced more early social withdrawal behavior at 3 months as compared with the full-term infants, and their mothers reported more symptoms of depression. As presently documented, at 12 months the mothers of the preterm children reported more child internalizing behavior than the mothers of the full-term children. Further, based on regression analyses, the results showed that early maternal depressive symptoms were a salient predictor of later child social emotional functioning, but no infant characteristics (neither withdrawal behavior, nor gestational age) bore a significant relation to child outcome. Hence, social withdrawal symptoms in lower risk 
moderately premature babies do not necessarily related relate to later problems in socio emotional functioning. However, the differences in strength of the relations between ADBB and EPDS, respectively, to the outcome at 12 months was modest. A clinical implication is that in work with infants and their mothers, clinicians should be aware of the complex interplay between signs of maternal postpartum depression and early infant withdrawal in planning ports of entry for early intervention. 


\section{References}

Aarnoudse-Moens, C. S., Weisglas-Kuperus, N., van Goudoever, J. B., \& Oosterlaan, J. (2009). Meta-analysis of neurobehavioral outcomes in very preterm and/or very low birth weight children. Pediatrics, 124, 717-728. 10.1542/ peds.2008-2816.

Bayley, N (2005). Bayley scales of infant and toddler development (3rd. ed.) San Antonio, TX: Pearson.

Berle, J. Ø., Aarre, T. F., Mykletun, A., Dahl, A. A., \& Holsten, F. (2003). Screening for postnatal depression: Validation of the Norwegian version of the Edinburgh Postnatal Depression Scale, and assessment of risk factors for postnatal depression. Journal of Affective Disorders, 76(1-3), 151-156. doi: 10.1016/s0165-0327(02)00082-4

Braarud, H. C., Slinning, K., Moe, V., Smith, L., Vannebo, U. T., Guedeney, A., \& Heimann, M. (2013). Relation between Social Withdrawal Symptoms in Full-Term and Premature Infants and Depressive Symtoms in Mothers: A Longitudinal Study. Infant Mental Health Journal, 34(6), 532-541. doi: Doi 10.1002/Imhj.21414

Burtchen, N., Alvarez-Segura, M., Mendelson, A.L., Dreyer, B.P., Castellanos, P.C., \& Guedeney, A. (2013). Screening for Sustained Social Withdrawal Behaviors in SixMonth-old Infants During Pediatric Primary Care Visits: Results From an At-risk Latino Immigrant Sample with High Rates of Maternal Major Depressive Disorder. Infant Mental Health Journal, 34(6), 532-552. doi: Doi 10.1002/Imhj.21414

Bhutta, A. T., Cleves, M. A., Casey, P. H., Cradock, M. M., \& Anand, K. J. (2002). Cognitive and behavioral outcomes of school-aged children who were born preterm: A metaanalysis. Journal of the American Medical Association, 288, 728-737. 10.1001/jama.288.6.728

Campbell, S.B., Cohn, J.F., \& Meyers, T. (1995). Depression in first-time mothers: Motherinfant interaction and depression chronicity. Developmental Psychology, 13, 349-357.

Carter, A. S., Briggs-Gowan, M. J., Jones, S. M., \& Littler, T. D. (2003). The Infant-Toddler Social and Emotional Assessment (ITSEA): Factor Structure, Reliability, and Validity. Journal of Abnormal Child Psychology, 31(5), 542-514.

Carter, A. S., Littler, C., Briggs-Gowan, M. J., \& Kogan, N. (1999). The infant-toddler social and emotional assessment (ITSEA): Comparing parent ratings to laboratory observations of task mastery, emotion regulation, coping behaviors, and attachment status. Infant Mental Health Journal, 20(4), 375-392. doi: Doi 10.1002/(Sici)10970355(199924)20:4<375::Aid-Imhj2>3.0.Co;2-P

Costa, R., \& Figueiredo, B. (2011). Infants' psychophysiological profile and temperament at 3 and 12 months. Infant Behavior \& Development, 34, 270-279.

Costa, R., \& Figueiredo, B. (2013). The Alarm Distress Baby Scale in a Longitudinal Portuguese Study Reanalyzed with Attachment Data. Infant Mental Health Journal, 34, 553-561.

Cox, J., Holden, J., \& Sagovsky, R. (1987). Detection of postnatal depression: Development of the 10 item Edinburgh Postnatal Depression Scale. British Journal of Psychiatry, $150,782-786$. 
Dollberg, D., Feldman, R., Keren, M., \& Guedeney, A. (2006). Sustained withdrawal behavior in clinic referred and non-referred infants. Infant Mental Health Journal, 27, 292-309.

Eberhard-Gran, M., Eskild, A., Tambs, K., Schel, B., \& Opjordsmoen, S. (2001). The Edinburgh Postnatal Depression Scale: Validation in a Norwegian community sample. Nordic Journal of Psychiatry, 55(2), 113-117.

Eberhard-Gran M., Slinning, K. \& Rognerud, M. (2014). Screening for postnatal depression a summary of current knowledge. Tidsskrift for den Norske Lageforening: Tidsskrift for Praktisk Medisin, 134, 297-301.

Field, T.M. (1995). Infants of depressed mothers. Infant behavior and Development, 18, 1-13.

Field, T.M. (1977). Effects of early separation, interaction deficits, and experimental manipulations in infant-mother face-to-face interaction. Child Development, 48, 763771.

Field, T.M. (2010). Postpartum depression effects on early interactions, parenting, and safety practices: A review. Infant Behavior and Development, 33, 1-6.

Gerhold, M., Laucht, M., Texdorf, C., Schmidt, M.H., \& Esser, G. (2002). Early motherinfant interaction as a precursor of childhood social withdrawal. Child Psychiatry and Human Development, 32, 277-293.

Goodman, S.H. (2007). Depression in mothers. In S. Nolen-Hoeksma, T.D. Cannon, \& T. Widiger (Eds.), Annual Review of Clinical Psychology, Vol. 3, pp. 107-135. Palo Alto: Annual reviews.

Goodman, S.H., Rouse, M.H., Connell, A.M., Broth Robbins, M., Hall, C.M. \& Heyward, D. (2011). Maternal depression and child psychopathology: A meta-analytic review. Clinical Child and Family Psychology Review, 14, 1-27.

Guedeney, A., \& Fermanian, J. (2001). A validity and reliability study of assessment and screening for sustained withdrawal reaction in infancy: The Alarm Distress Baby Scale. Infant Mental Health Journal, 22, 559-575.

Guedeney, A., Marchand-Martin, L., Cote, S.J., Larroque,B.,\& the EDEN Mother-Child Cohort Study Group. (2012). Perinatal risk factors and social withdrawal behavior. European Child \& Adolescent Psychiatry,21, 185-191.

Guedeney, A., Matthey, S., \& Puura, K. (2013). Social withdrawal beahvior in infancy: A history of the concept and a review of published studies using the Alarm Distress baby Scale. Infant mental Health Journal, 34, 516-531.

Izard, C. E., Huebner, R. R., Risser, D., McGinness, G., \& Dougherty, L. (1980). The young infant's ability to produce discrete emotional expressions. Developmental Psychology, $16,132-140$.

Lewis, M. (2014). The Rise of Consciousness and the Development of Emotional Life. New York/London: Guilford Press

Luby, J.L., Gaffrey, M.S, Tillman, R., April, L.M. \& Belden, A.C (2014). Trajectories of Preschool Disorders to full DSM depression at school age and early adolescence: Continuity of preschool depression. American Journal of Psychiatry, 171, 768-776.

Matthey, S., Guedeney, A., Starakis, N., \& Barnett, B. (2005). Assessing the social behavior of infants: Use of the ADBB scale and relationship to mother's mood. Infant Mental Health Journal, 26, 442-458. 
Milne, L., Greenway, P., Guedeney, A., \& Larroque, A. (2009). Long term developmental impact of social withdrawal in infants. Infant Behavior \& Development, 32, 159-166.

O’Hara, M.W., \& Swain, A.M. (1996). Rates and risk of postpartum depression: A metaanalysis. International Review of Psychiatry, 8, 37-54.

Puura, K., Guedeney, A., Mäntymaa, M., \& Tamminnen, T. (2007). Detecting infants in need: Are complicated measures really necessary?. Infant Mental Health Journal, 28, 409421.

Reynold, C.R. \& Kamphaus, S.W. (2004). BASC-2 behavior assessment system for children (2nd ed.). Circle Pines, MN: American Guidance Service.

Salomonsson, B., \& Sleed, M. (2010). The Ages \& Stages Questionnaire: Social-Emotional: A validation study of a mother-report questionnaire on a clinical mother-infant sample. Infant Mental Health Journal, 31(4), 412-431. doi: 10.1002/imhj.20263

Sameroff, A.J. (2010). A unified theory of development: A dialectic integration of nature and nurture Child Development 81, 6-22.

Sameroff, A.J., \& McKenzie, M.J. (2003). Research strategies for capturing transactional models of development: The limits of the possible. Development and Psychopathology, 15, 613-640.

Schothorst, P.F., \& van Engeland, H. (1996). Long-term behavioral sequelae of prematurity. Journal of the American Academy of Child \& Adolescent Psychiatry, 35, 175-183

Stein, A., Pearson, R. M., Goodman, S. H., Rapa, E., Rahman, A., McCallum, M., Howard, L.M., Pariante, C. M. (2014). Effects of perinatal mental disorders on the fetus and child. The Lancet, 384, 1800-1819.

Sykes, D.H., Hoy, E.A., Bill, J.M., McLure, B.G., Halliday, H.L., \& McReid, M. (1997). Behavioral adjustment in school of very low birthweight children. Journal of Child Psychology and Psychiatry, 38, 315-325.

Squires, J., Bricker, D., \& Twombly, E. (2002). Ages and Stages Questionnaires: SocialEmotional (ASQ: SE): Baltimore.

Tessier, R., Nadeau, L., Boivin, M., \& Tremblay, R.E. (1997). The social behavior of 11-to 12-year old children born as low birthweight and/or premature infants. International Journal of Behavioral Development, 21, 795-811. 
Table 1: Descriptive statistics for independent, dependent and control variables for each separate group full-term and preterm), and group differences

\begin{tabular}{|c|c|c|c|c|c|c|c|c|}
\hline & Full- & & & emature & & & & \\
\hline Variable & $\mathrm{N}$ & $\mathrm{M}(\mathrm{SD})$ & Range & $\mathrm{N}$ & $\mathrm{M}(\mathrm{SD})$ & Range & $t$ & $p$ \\
\hline GA (weeks) & 227 & $39.93(1.09)$ & $37.3-42.4$ & 64 & $34.73(1.70)$ & $\begin{array}{l}30.0- \\
36.9\end{array}$ & 23.123 & $<0.001$ \\
\hline $\begin{array}{l}\text { Birth weight } \\
\text { (grams) }\end{array}$ & 238 & $3625.50(473.68)$ & $\begin{array}{l}2250.0- \\
5580.0\end{array}$ & 64 & $\begin{array}{l}2353.16 \\
(498.20)\end{array}$ & $\begin{array}{l}1220.0- \\
3660.0\end{array}$ & 18.867 & $<0.001$ \\
\hline Maternal age & 237 & $30.08(4.93)$ & $16-46$ & 64 & $30.80(5.26)$ & $22-42$ & -1.023 & 0.307 \\
\hline ADBB $3 \mathrm{~m}^{\mathrm{a}}$ & 238 & $0.89(1.52)$ & $0-9$ & 62 & $1.58(2.12)$ & $0-9$ & -2.417 & 0.018 \\
\hline EPDS $3 \mathrm{~m} .^{a}$ & 221 & $3.75(3.29)$ & $0-20$ & 61 & $5.13(4.47)$ & $0-17$ & -2.254 & 0.027 \\
\hline $\begin{array}{l}\text { ITSEA Int. } \\
\text { domain } 12 \mathrm{~m} \text {. }\end{array}$ & 182 & $44.86(9.45)$ & $28-70$ & 56 & $48.23(9.88)$ & $30-65$ & -2.313 & 0.022 \\
\hline $\begin{array}{l}\text { ITSEA Ext. } \\
\text { domain } 12 \mathrm{~m} .\end{array}$ & 178 & $47.34(8.20)$ & $32-72$ & 56 & $47.80(7.67)$ & $35-72$ & -0.372 & 0.710 \\
\hline $\begin{array}{l}\text { ITSEA Dysr. } \\
\text { domain } 12 \mathrm{~m} \text {. }\end{array}$ & 185 & $41.97(12.39)$ & $21-89$ & 56 & $\begin{array}{l}42.93 \\
(12.79)\end{array}$ & $24-71$ & -0.505 & 0.614 \\
\hline $\begin{array}{l}\text { ASQ:SE } 12 \\
\mathrm{~m} .\end{array}$ & 190 & $14.97(12.40)$ & $0-60$ & 48 & $15.31(9.97)$ & $0-40$ & -0.175 & 0.861 \\
\hline
\end{tabular}


Note. GA: gestational age; ADBB: Alarm Distress Baby Scale, EPDS: Edinburgh Postnatal Depression Scale; ITSEA: Infant Toddler Social Emotional Assessment, Int.: Internalizing domain, Ext.: Externalizing domain, Dysr.: Dysregulation domain, ASQ:SE: Ages and Stages

Questionnaires: Social Emotional

${ }^{a}$ ADBB 3 months: Median 0, quartiles 0 and 1. EPDS 3 months: median 3, quartiles 1 and 5.

p-values based on permutation t-tests (100000 permutations) were 0.025 for ADBB and 0.030 for EPDS at 3 months. 
Table 2: Descriptive statistics for the dependent variables for the two groups combined

Means, SDs, and Range for independent, dependent and control variables for the two groups combined

\begin{tabular}{|c|c|c|c|}
\hline Variables & $\mathrm{N} \quad(\%)$ & $\mathrm{M}(\mathrm{SD})$ & Range \\
\hline Birth weight (g) & 302 & $3355.86(707.02)$ & $1220.0-5580.0$ \\
\hline GA (weeks) & 291 & $38.78(2.49)$ & $30.0-42.4$ \\
\hline Age of mother (year) & 301 & $30.23(5)$ & $16-46$ \\
\hline Mothers education (\%) & 297 & & \\
\hline 9 years or less & $17(5.7)$ & & \\
\hline High school & $73(24.6)$ & & \\
\hline 1-3 years of college/univ. & $155(52.2)$ & & \\
\hline More than 3 years univ. & $52(17.5)$ & & \\
\hline EPDS 3 month & 282 & $4.05(3.62)$ & $0-20$ \\
\hline ADBB 3 month & 300 & $1.03(1.69)$ & $0-9$ \\
\hline \multicolumn{4}{|l|}{ ITSEA 12 months: } \\
\hline Externalising & 234 & $47.45(8.06)$ & $32-72$ \\
\hline Internalising & 238 & $45.65(9.64)$ & $28-70$ \\
\hline Dysregulation & 241 & $42.19(12.46)$ & $21-89$ \\
\hline ASQ: SE 12 months & 238 & $15.04(11.93)$ & $0.0-60.0$ \\
\hline
\end{tabular}

Note. EPDS: Edinburgh Postnatal Depression Scale; ADBB: Alarm Distress Baby Scale; ITSEA: Infant Toddler Social Emotional Scale; ASQ: SE: Ages and Stages Questionnaire 
Table 3: Multiple linear regression analyses of the relation between symptoms of maternal depression and infant social withdrawal at 3 months and child social emotional development at 12 months $(\mathrm{N}=208-214$ depending on the outcome measure).

\begin{tabular}{|c|c|c|c|c|c|c|c|c|c|}
\hline \multicolumn{6}{|c|}{ Model for ITSEA Externalizing domain: } & \multicolumn{4}{|c|}{ Model for ITSEA Internalizing domain: } \\
\hline & Coeff. & Lower bound & Upper bound & $\mathrm{p}$ & & Coeff. & Lower bound & Upper bound & $\mathrm{p}$ \\
\hline EPDS 3 months & 0.37 & 0.06 & 0.67 & 0.018 & EPDS 3 months & 0.45 & 0.07 & 0.84 & 0.020 \\
\hline ADBB 3 months & -0.36 & -0.94 & 0.21 & 0.214 & ADBB 3 months & -0.35 & -1.09 & 0.39 & 0.348 \\
\hline \multicolumn{6}{|c|}{ Model for ITSEA Dysregulation domain: } & \multicolumn{3}{|c|}{ Model for ASQ: SE: } & \\
\hline EPDS 3 months & 0.55 & 0.05 & 1.05 & 0.030 & EPDS 3 months & 0.60 & 0.11 & 1.10 & 0.017 \\
\hline ADBB 3 months & -0.14 & -1.11 & 0.83 & 0.776 & ADBB 3 months & 0.35 & -0.66 & 1.36 & 0.499 \\
\hline
\end{tabular}

Note. Coeff.: Regression coefficients. All models are adjusted for level of maternal education, maternal age and infant gestational age. Lower and upper bounds: for $95 \%$ confidence intervals.

Variance inflation factors (VIF): ITSEA Externalizing domain: VIF $\leq 2.00$, ITSEA Internalizing domain: VIF $\leq 1.96$, ITSEA Dysregulation domain: $\mathrm{VIF} \leq 1.98$, ASQ:SE: VIF $\leq 1.90$. 